\title{
Costo por estadio de la atención integral del paciente diabético tipo 2 con enfermedad renal crónica
}

\author{
I ${ }^{1}$ Enrique Villarreal Ríos, ${ }^{2}$ Rosaura Padilla Anaya, ${ }^{3}$ Emma Rosa Vargas Daza, \\ ${ }^{4}$ Lidia Martínez González, ${ }^{5}$ Liliana Galicia Rodríguez I
}

Resumen: Objetivo: Identificar el costo de la enfermedad renal crónica por estadio en pacientes con diabetes. Métodos: Estudio de costos en diabéticos tipo 2, se integraron cinco grupos definidos a partir del estadio de la enfermedad renal crónica. Se incluyeron aquellos con más de cinco años de evolución de la diabetes tipo 2 , el tamańo de la muestra se calculó para cada estadio y la selección fue aleatoria simple. El diagnóstico por estadio se calculó mediante la ecuación de Cockcroft-Gault. El costo promedio se integró a partir del perfil de uso y el costo unitario, se realizó estimación de la proyección del costo, también se realizó estimación del costo para un paciente con 25 ańos de vida con enfermedad renal crónica. Resultados: En el estadio 1 el costo anual es $\$ 587.39$, y en el estadio 5, $\$ 10,748.51$.

Otorgar la atención durante 25 años en una población de 100 pacientes diabéticos ajustados por letalidad, permanencia en el estadio e inflación cuesta $\$ 7,067,674$ y el costo promedio de un individuo en este mismo escenario es $\$ 70,672$. Conclusión: Se puede decir que el costo por estadio de la enfermedad renal crónica en el paciente diabético es alto con notable diferencia entre los distintos estadios.

> Palabras-clave: diabetes mellitus; insuficiencia renal crónica; costos.

\author{
1 Instituto Mexicano del Seguro \\ Social. Querétaro, Mexico \\ (evr57@hotmail.com). \\ 2 Instituto Mexicano del Seguro \\ Social. Querétaro, Mexico \\ (rouse0308@hotmail.com). \\ ${ }^{3}$ Instituto Mexicano del Seguro \\ Social. Querétaro, Mexico \\ (emma.vargas@imss.gob.mx). \\ ${ }^{4}$ Instituto Mexicano del Seguro \\ Social. Querétaro, Mexico (lidia. \\ martinez@imss.gob.mx). \\ ${ }^{5}$ Instituto Mexicano del Seguro \\ Social. Querétaro, Mexico \\ (liliana.galicia@imss.gob.mx).
}

Recebido em: 17/12/2015

Aprovado em: 08/09/2017 


\section{Introducción}

La diabetes tipo 2 constituye una verdadera epidemia (INSTITUTO MEXICANO..., 2012) y el costo del tratamiento de las complicaciones crónicas representa la mitad de los costos de atención de la diabetes (ARREDONDO; DAMIAN; DE ICAZA, 1995; ADA, 2012. Es la primera causa de enfermedad renal crónica (INSTITUTO MEXICANO..., 2009), de acuerdo a los criterios de la guía K/DOQUI se entiende por enfermedad renal crónica la presencia de lesión renal con o sin descenso del filtrado glomerular durante un periodo de tiempo igual o superior a 3 meses (NATIONAL KIDNEY FOUNDATION, 2002; 2006).

La National Kidney Foundation-Kidney Disease Outcomes Quality Initiative (US NKF-KDOQI) clasifica la enfermedad renal crónica en cinco etapas, determinadas por la velocidad de filtración glomerular estimada con ecuaciones de predicción como Cockcroft-Gault o MDRD (GRACÍA, 2006; LEVEY, 2008).

El número de pacientes con enfermedad renal crónica se ha incrementado en todo el mundo (LEVEY, 2007) y existen reportes que seńalan la perdida de la función renal en estadios tempranos (ALCÁZAR, 2008), consecuentemente la necesidad de procedimientos de diálisis se ha incrementado y con ello el costo de atención (ARREDONDO; RANGEL; DE ICAZA, 1998).

Se ha estimado que el costo anual promedio por paciente en el estadio 1 es $€ 32,000$, estadio $2 € 38,764$, estadio $3 € 33,144$, estadio $4 € 41,928$ y $€ 42,000$ en el estadio 5 (GÓRRIZ, 2008). También se ha señalado que los pacientes con ERC en estadio del 1 al 4 constituyen el 3.3\% de la población general atendida y consumen entre el 5.5\% y el 8\% del presupuesto total (GARRIDO, 2007; 2008). En este contexto, el objetivo del artículo es identificar el costo de la atención integral del paciente diabético tipo 2 con enfermedad renal crónica por estadio.

\section{Métodos}

Se realizó un estudio de costos en pacientes diabéticos tipo 2 pertenecientes a tres unidades de Medicina Familiar del Instituto Mexicano del Seguro Social en la ciudad de Querétaro, México, el periodo de estudio comprendió un año. 
Se integraron cinco grupos de estudio definidos en función del nivel de filtración glomerular estimado mediante la ecuación de Cockcroft-Gault utilizando la clasificación de National Kidney Foundation (K/DOQI):

- Grupo 1. Filtración glomerular mayor de $90 \mathrm{ml}$ por minuto por 1,73 m2;

- Grupo 2. Filtración glomerular de 60 a $89.9 \mathrm{ml}$ por minuto por 1,73 m2;

- Grupo 3. Filtración glomerular de 30 a $59.9 \mathrm{ml}$ por minuto por 1,73 m2;

- Grupo 4. Filtración glomerular de 15 a $29.9 \mathrm{ml}$ por minuto por 1,73 m2;

- Grupo 5. Filtración glomerular menor de $15 \mathrm{ml}$ por minuto por 1,73 m2.

Se incluyeron a todos aquellos pacientes con más de cinco años de evolución de diabetes tipo 2; se excluyeron lo que presentaron obesidad mórbida, desnutrición, diabetes tipo 1, litiasis renal, poliquistosis renal, enfermedades autoinmunes, infecciones recurrentes del tracto urinario y aquellos en los que se diagnosticó hipertensión arterial antes de diabetes; y se eliminaron los sometidos a hemodiálisis. El tamańo de la muestra se calculó para cada estadio con la fórmula de promedios para población finita, con 95\% de confianza $(Z=1.64)$, considerando el cuadro 1 , a seguir.

Cuadiro 1. Tamańo de la muestra por cada estadio

\begin{tabular}{|c|c|c|c|}
\hline Estadio & $\begin{array}{c}\text { Total de pacientes } \\
\text { existentes }\end{array}$ & $\begin{array}{c}\text { Costo promedio (euros) } \\
\text { (Hipótesis) }\end{array}$ & $\begin{array}{c}\text { Total de la } \\
\text { muestra }\end{array}$ \\
\hline Estadio 1 & 480 & 32.000 & 59 \\
\hline Estadio 2 & 430 & 38.764 & 58 \\
\hline Estadio 3 & 526 & 33.144 & 61 \\
\hline Estadio 4 & 50 & 41.928 & 29 \\
\hline Estadio 5 & 50 & 42.000 & 29 \\
\hline
\end{tabular}

Elaborado por los autores.

La técnica muestral fue aleatoria simple, considerando como marco muestral el censo de pacientes con diabetes tipo 2, a partir de este listado se identificó edad, peso y creatinina para establecer el diagnóstico de enfermedad renal crónica integrando los pacientes a cada estadio. Cuando el sujeto de estudio no 
cumplía los criterios de selección se tomó el inmediato siguiente. Las variables estudiadas incluyeron:

- Sociodemográficas (edad, sexo, escolaridad);

- De salud (años de evolución de DM2, años de evolución de hipertensión arterial, nivel de glucosa, peso, talla y creatinina);

- El diagnóstico por estadio se integró para hombres y mujeres con la fórmula de Cockcroft-Gault

\begin{tabular}{|c|c|c|}
\hline Hombres & Mujeres & \\
\hline$(140-$ edad $) *$ peso & {$\left[(140-\text { edad })^{*}\right.$ peso $]$} & \multirow{2}{*}{ * 0.85} \\
\hline $72 *$ creatinina sérica & {$[72 *$ creatinina sérica] } & \\
\hline
\end{tabular}

- Costo promedio anual: se estimó para la atención ambulatoria de medicina familiar, nutrición, nefrología, cardiología, oftalmología y medicina interna; se complementó con número de hospitalizaciones y colocación de catéter de diálisis; al igual que laboratorio, medicamentos y manejo de la peritonitis. El costo promedio anual se dividió en costo promedio fijo anual y el costo promedio variable anual. El cálculo se integró a partir de perfil de uso de los servicios de salud y los costos unitarios de cada uno de ellos.

$>$ Perfil de uso anual: se identificó el número de veces que utilizó los servicios mencionados en el período de un año;

$>$ Costo unitario: se integró a partir del costo unitario fijo y el costo unitario variable.

- El costo unitario fijo se estimó con la técnica de tiempos y movimientos considerando el sueldo nominal del personal de salud que tiene contacto directo con el paciente, el personal de apoyo directivo y administrativo; los servicios elementales para otorgar la atención; el equipo, mobiliario e infraestructura. El costo se estimó por minuto y posteriormente se multiplicó por el promedio de minutos destinados a la atención de cada paciente.;

- Para el costo unitario variable se utilizó la técnica de microcosteo recabando en el expediente clínico la cantidad y tipo de medicamentos y estudios de laboratorio empleados por el paciente. 


\begin{tabular}{|l|l|l|}
\hline $\begin{array}{l}\text { Costo } \\
\text { unitario } \\
\text { fijo }\end{array}$ & $=$ & $\begin{array}{l}\text { Sueldo personal operativo + Sueldo personal } \\
\text { administrativo + Sueldo personal directivo }+ \\
\text { Servicios elementales } \\
+ \text { Equipo + Mobiliario + Infraestructura }\end{array}$ \\
\hline $\begin{array}{l}\text { Costo } \\
\text { unitario } \\
\text { variable }\end{array}$ & $=$ & $\begin{array}{l}\text { Medicamentos de primer nivel } \\
+ \text { Medicamentos de segundo nivel } \\
+ \text { Tratamiento de la peritonitis } \\
+ \text { Estudios de laboratorio }\end{array}$ \\
\hline \begin{tabular}{|l|l|l|} 
Costo \\
unitario
\end{tabular} & $=$ & Costo unitario fijo + Costo unitario variable \\
\hline Costo promedio & $=$ & Perfil de uso * Costo unitario \\
\hline
\end{tabular}

- Estimación de la proyección del costo para 100 pacientes y costo promedio.

La proyección se realizó para una población de 100 pacientes diabéticos que debutan en el estadio 1;

El tiempo de vida del paciente con enfermedad renal crónica es de 25 años a partir de que se hace el diagnóstico del estadio 1;

La letalidad se obtuvo de lo reportado en la literatura y se aplicó por año a la población que permanecía con vida;

La población viva para el año siguiente es el resultante de aplicar la letalidad a la población que permanecía con vida el año anterior;

El porcentaje de población por estadio y años de evolución de la enfermedad renal crónica se obtuvo de la población entrevistada;

> El total de población por estadio y tiempo de evolución se obtuvo de multiplicar el total de población viva en el año respectivo por el porcentaje de población en cada estadio;

La proyección del costo unitario anual por estadio se obtuvo del cálculo realizado en este trabajo multiplicado por la tasa de inflación reportada por el Banco de México (7\%);

El costo por estadio y tiempo de evolución se estimó multiplicando el total de población por tiempo de evolución y estadio por el costo anual por estadio y tiempo de evolución; 
> El costo total para la población original planteada se obtuvo de la suma de los costos totales por año de evolución y estadio;

> El costo promedio por individuo a lo largo de la vida se obtuvo al dividir el costo total para la población entre el total de la población original.

- Estimación de la proyección del costo en un paciente con enfermedad renal crónica en estadio 1 que progresa a estadio 5 en 25 años.

> Se definieron los años de permanencia en cada estadio;

> Se identificó el costo unitario por estadio y año ajustado por inflación;

> Se sumó el costo unitario anual por año y por estadio a 25 años.

El análisis estadístico se realizó con promedios, porcentajes y proyecciones.

\section{Resultados}

En todos los estadios el tiempo promedio de evolución de la diabetes tipo 2 es superior a los 10 años, y corresponde al estadio 5 el tiempo de evolución más alto, 21.21 años. Contrario al comportamiento de esta variable, el peso más alto se identifica en el estadio 1. En la tabla 1 se presentan las características sociodemográficas y de salud por estadio.

Tabla 1. Características sociodemográficas y de salud por estadio

\begin{tabular}{lccccc}
\hline & Estadio 1 & Estadio 2 & Estadio 3 & Estadio 4 & Estadio 5 \\
\hline Sociodemográficas & & & Porcentajes & & \\
Sexo (Femenino) & 62.3 & 47.3 & 50.8 & 63.3 & 41.4 \\
Escolaridad (Primaria o menos) & 61.3 & 63.6 & 65.6 & 76.3 & 58.6 \\
\hline Características de salud & & & Promedios & & \\
Edad (años) & 58.21 & 61.25 & 66.38 & 64.10 & 60.97 \\
Evolución de la DM2 (años) & 10.02 & 12.29 & 14.85 & 16.57 & 21.21 \\
Evolución de la HTA (años) & 3.80 & 5.51 & 6.36 & 10.37 & 8.45 \\
Peso (kilogramos) & 77.66 & 73.92 & 65.86 & 59.97 & 63.61 \\
Talla (centímetros) & 157.44 & 159.75 & 157.07 & 156.67 & 159.69 \\
Creatinina (mg/dl) & 0.76 & 1.00 & 1.33 & 2.19 & 6.80 \\
Promedio de glucosa (mg/dl) & 196.20 & 180.20 & 169.30 & 137.44 & 108.40 \\
\hline
\end{tabular}


El uso de los servicios de salud reporta una mayor intensidad en los estadios

4 y 5; ésta misma tendencia se observa en el costo promedio anual, en el estadio $4 \$ 178.67$ y en el estadio 5 se reporta $\$ 987.31$. En la tabla 2 se presentan el perfil de uso anual, el costo unitario fijo y el costo promedio fijo anual por estadio y por tipo de servicio.

El costo promedio variable anual por medicamentos en primer nivel, segundo nivel y tratamiento de la peritonitis en el estadio $5(\$ 9,154.45)$ es mayor al observado en el estadio 1 (\$483.22). En la tabla 3 se presenta el costo por estadio y tipo de medicamento.

El costo promedio variable anual de estudios de laboratorio es $\$ 304.29$ en el estadio 5; en esta cantidad se incluye el costo de primer nivel, segundo nivel y manejo de la peritonitis; cantidad superior reportada en cualquiera de los otros estadios. En la tabla 4 se presenta el costo promedio variable anual de estudios de laboratorio por estadio.

El costo promedio total en el estadio 1 (\$587.39) y estadio 2 (\$584.47) es muy semejante, y este mismo comportamiento se aprecia en el estadio $3(\$ 1,328.02)$ y estadio 4 (\$1,481.48), se incrementa considerablemente en el estadio $5(\$ 10,748.51)$. En la tabla 5 se presenta el costo total de la atención integral por estadio.

La proyección del costo a 25 años en una población de 100 pacientes que debutan con estadio 1 de enfermedad renal crónica es $\$ 7,067,674$ y el costo promedio por paciente es $\$ 70,677$. En la tabla 6 se presenta el costo por estadio y tiempo de evolución.

El costo de un individuo en estadio 1 y su seguimiento a 25 años con progresión al estadio 5 es $\$ 115,748$. 


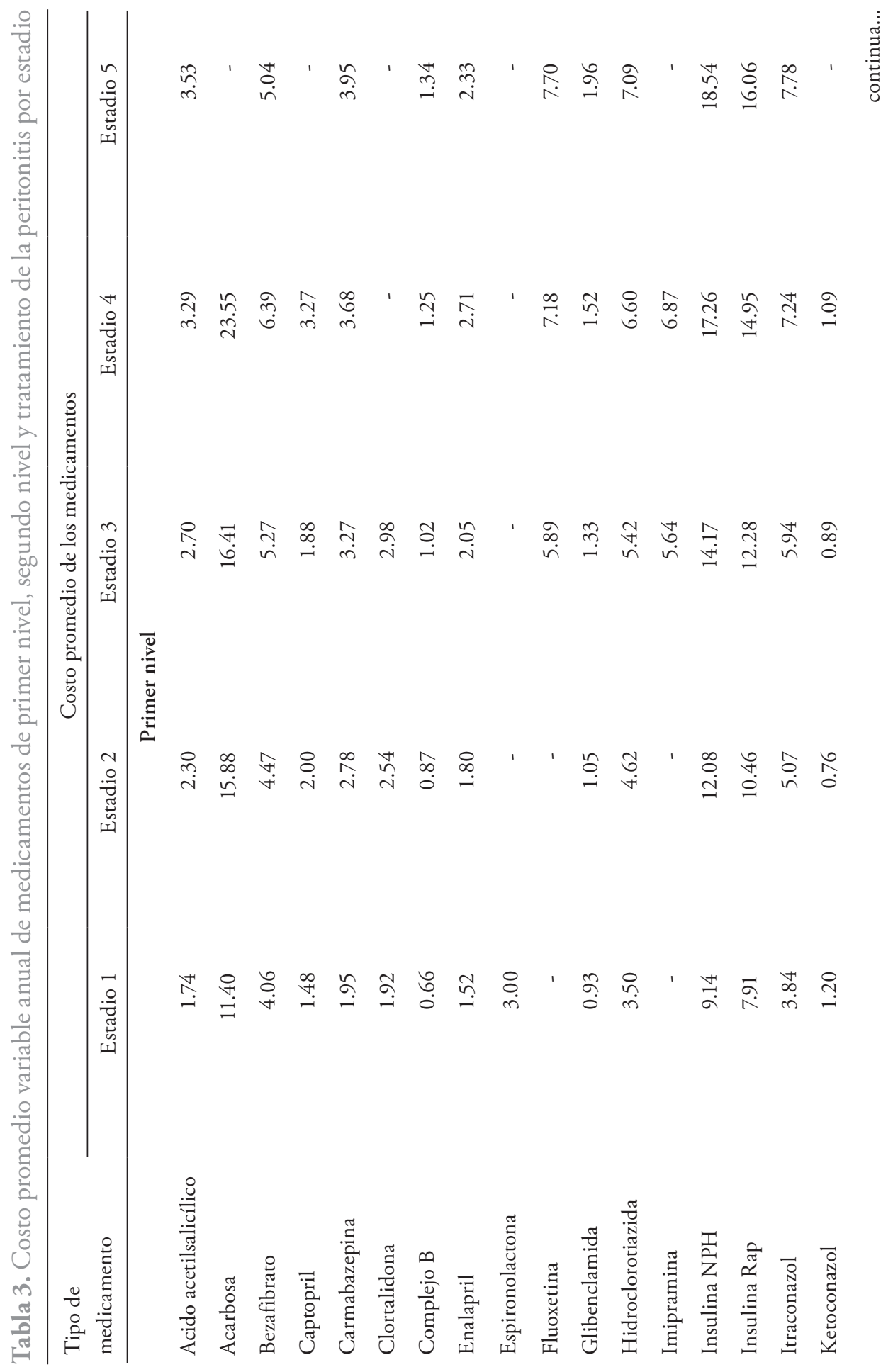


1134

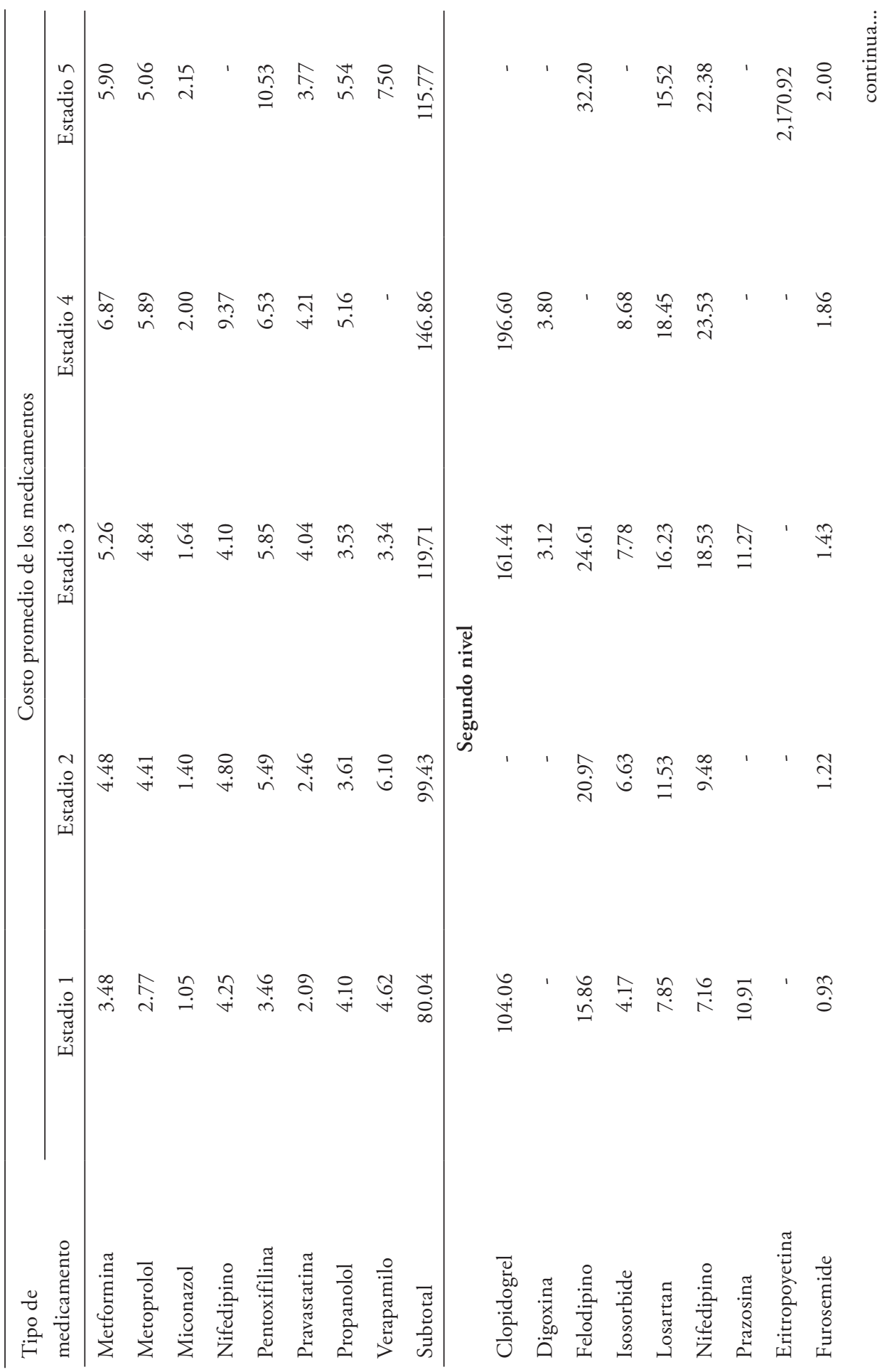




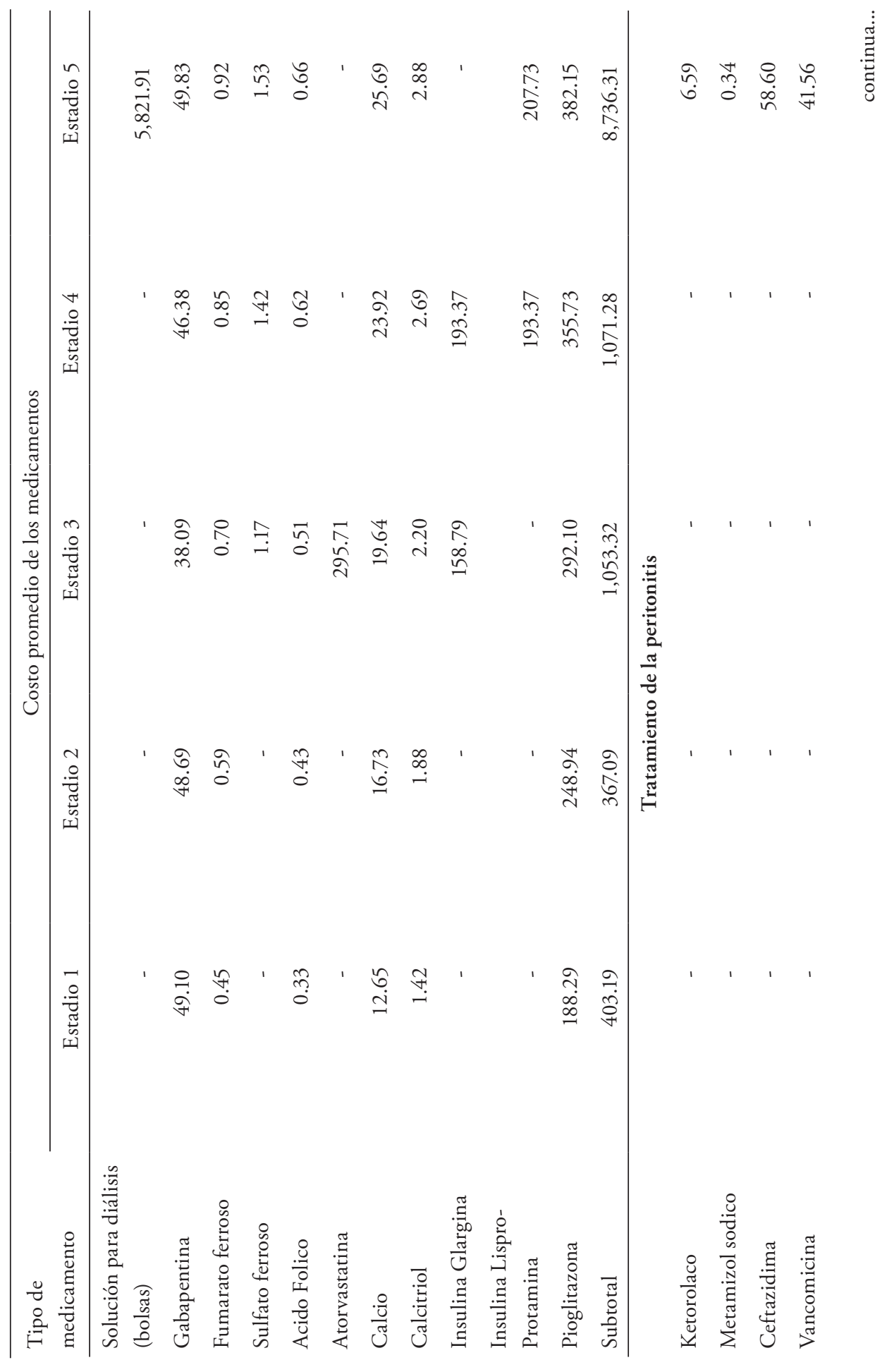




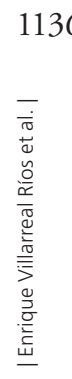

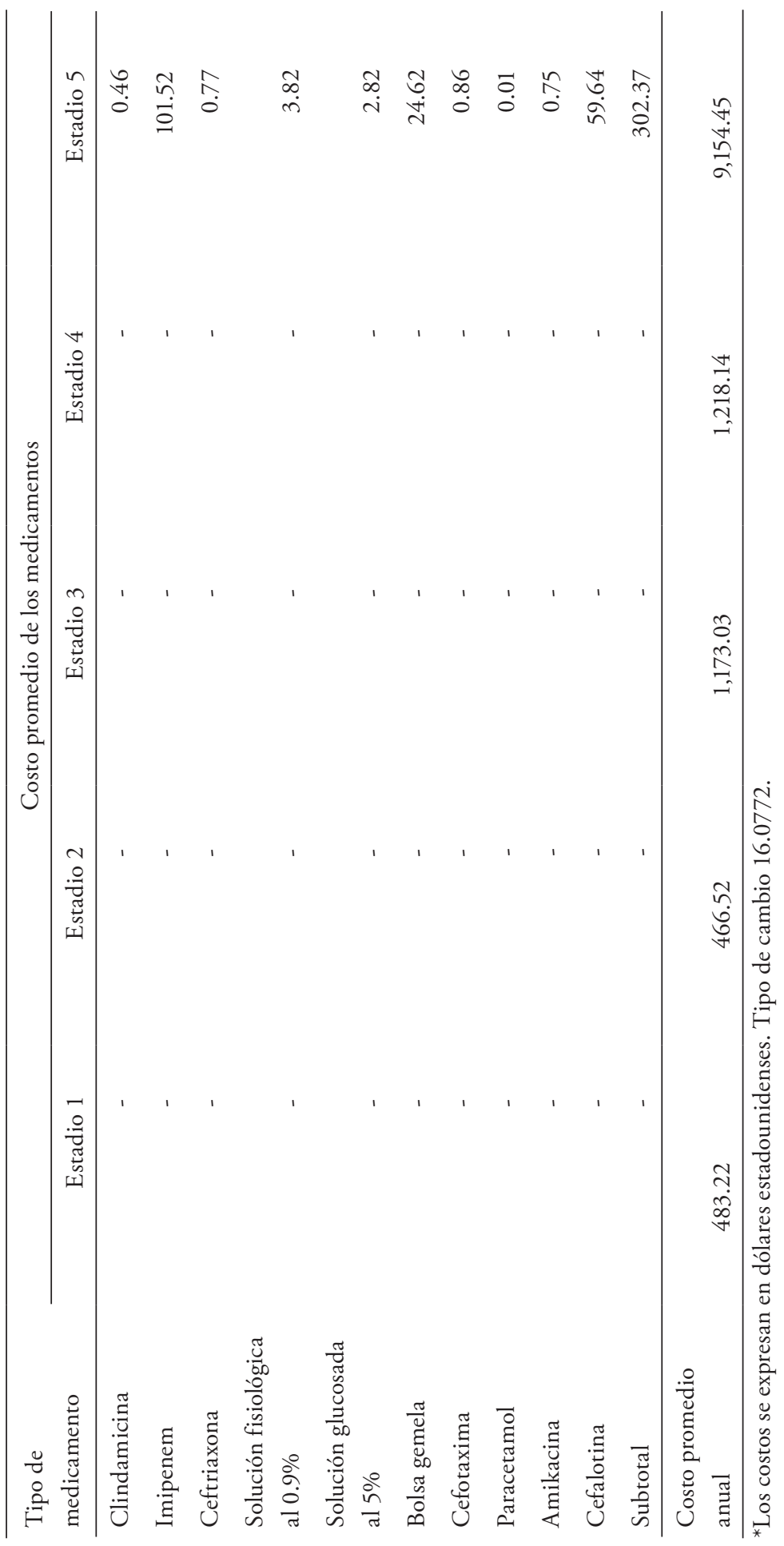

Physis Revista de Saúde Coletiva, Rio de Janeiro, 27 [ 4 ]: 1125-1146, 2017 


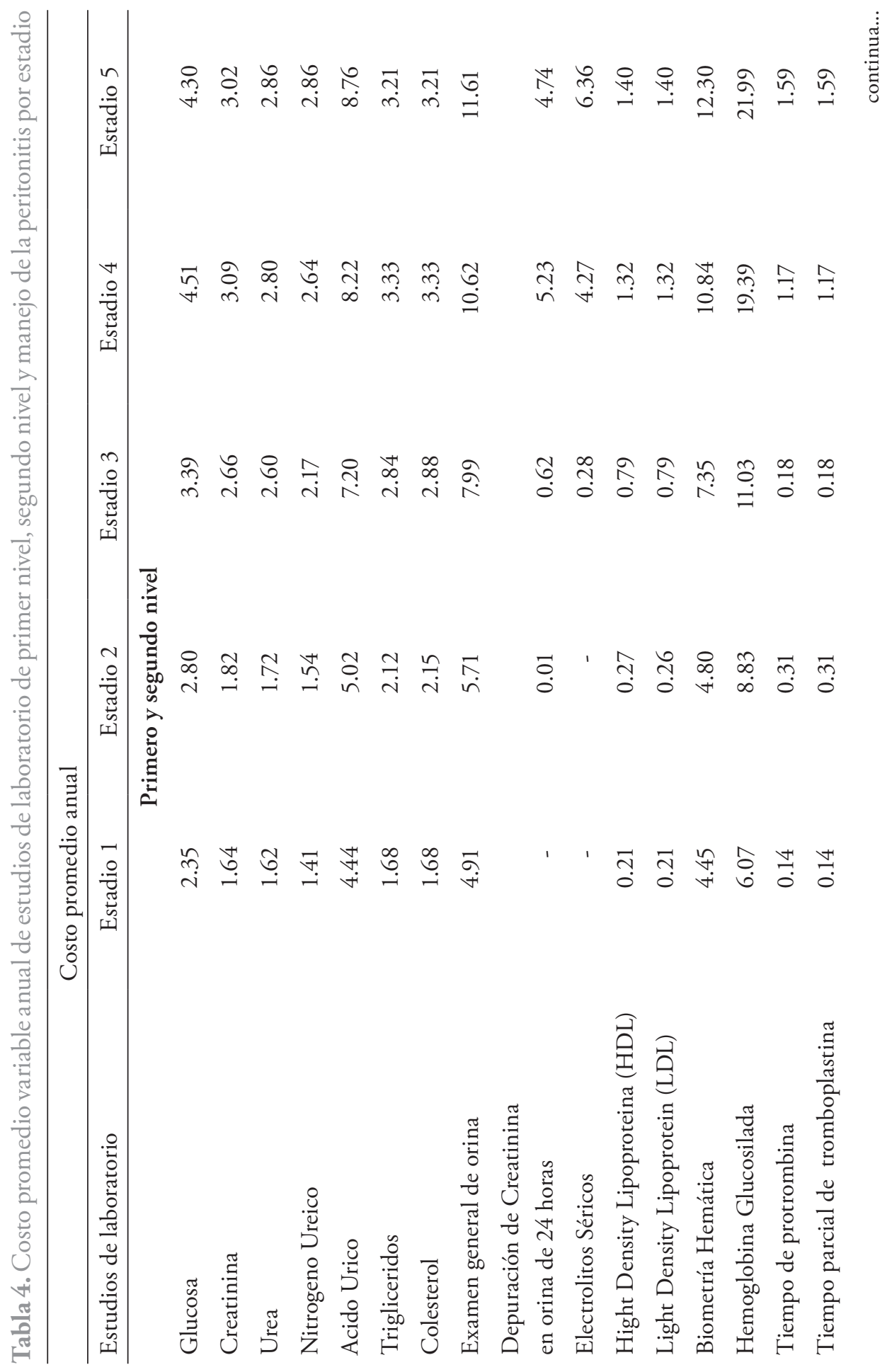


1138

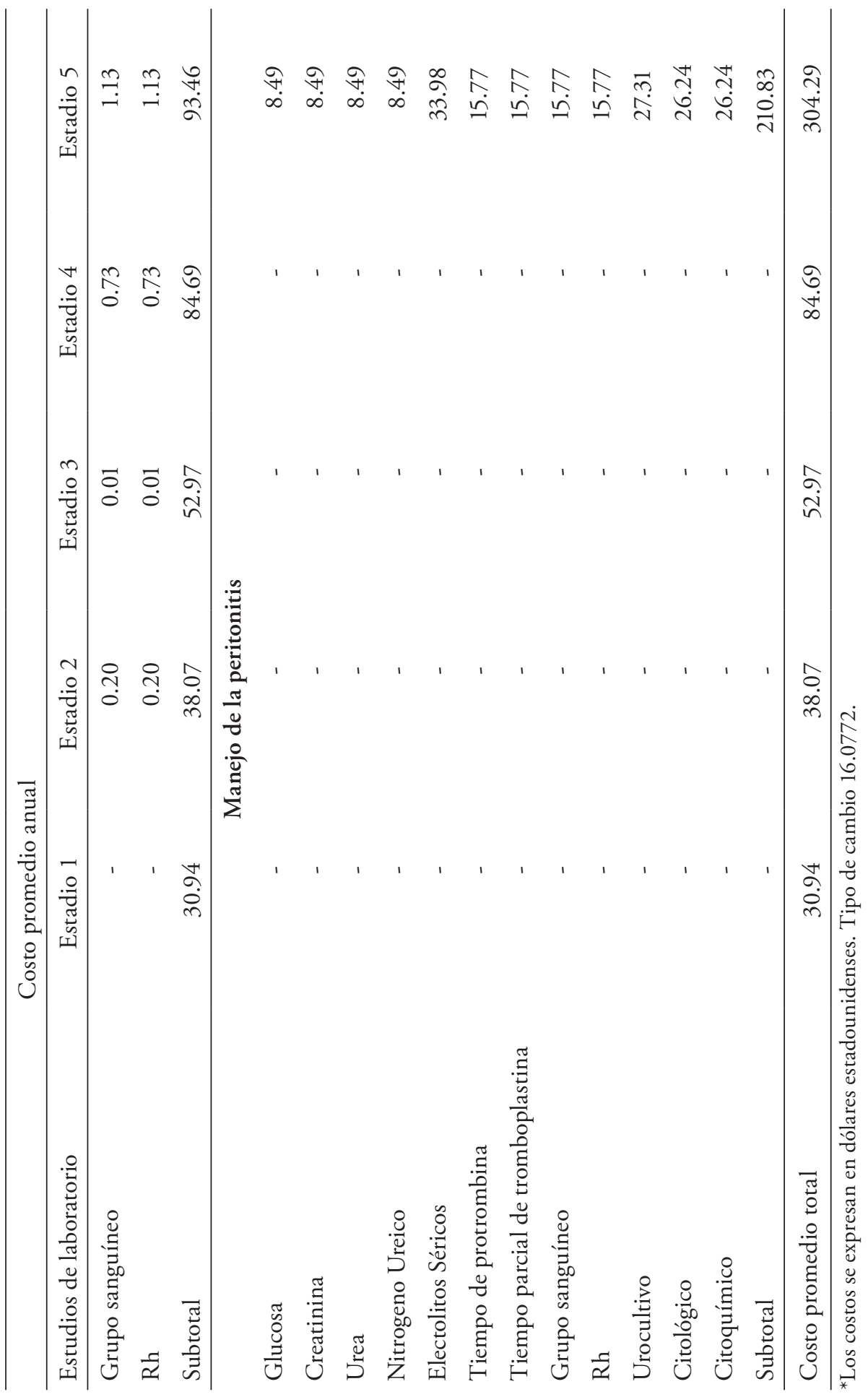


Tabla 5. Costo promedio total de la atención integral por estadio

Costo total por estadio

\begin{tabular}{lccccc}
\hline Servicios & Estadio 1 & Estadio 2 & Estadio 3 & Estadio 4 & Estadio 5 \\
Consulta externa & 42.27 & 58.59 & 79.06 & 107.09 & 121.09 \\
Urgencias & 24.87 & 12.37 & 17.95 & 57.90 & 203.68 \\
Hospital & 6.11 & 8.82 & 4.89 & 13.68 & 65.59 \\
Colocación de catéter de diálisis & - & - & - & - & 93.60 \\
Laboratorio & 30.93 & 38.15 & 53.08 & 84.69 & 93.44 \\
Medicamentos & 483.21 & 466.53 & $1,173.04$ & $1,218.11$ & $9,154.51$ \\
Peritonitis urgencias & - & - & - & - & 162.68 \\
Peritonitis hospital & - & - & - & - & 340.67 \\
Peritonitis laboratorio & - & - & - & - & 210.83 \\
Peritonitis medicamentos & - & - & - & - & 302.42 \\
\hline Costo promedio total de la & & & & & \\
atención integral & 587.39 & 584.47 & $1,328.02$ & $1,481.48$ & $10,748.51$ \\
\hline
\end{tabular}

${ }^{*}$ Los costos se expresan en dólares estadounidenses. Tipo de cambio 16.0772.

Tabla 6. Proyección del costo en 100 pacientes por estadio y tiempo de evolución de la enfermedad renal crónica, ponderado por inflación, letalidad y permanencia en el estadio

\begin{tabular}{cccccc}
\hline $\begin{array}{c}\text { Tiempo de evolución } \\
\text { de la ERC (años) }\end{array}$ & Estadio 1 & Estadio 2 & Estadio 3 & Estadio 4 & Estadio 5 \\
\hline 1 & 29,370 & 19,482 & 22,134 & - & - \\
2 & 31,111 & 20,638 & 23,446 & - & - \\
3 & 19,774 & 32,792 & 29,804 & - & - \\
4 & 36,748 & 18,283 & 16,616 & 9,268 & 67,244 \\
5 & 34,805 & 8,658 & 49,181 & 21,946 & - \\
6 & 36,737 & 12,185 & 36,914 & 20,590 & 74,693 \\
7 & 25,023 & 28,011 & 56,574 & - & 57,236 \\
& 32,799 & 13,054 & 44,492 & 33,089 & 120,036 \\
& & & & & continua...
\end{tabular}


Tiempo de evolución de la ERC (años)

\begin{tabular}{cccccc}
\hline 9 & 19,162 & 44,489 & 28,882 & 16,109 & 116,879 \\
10 & 23,442 & - & 79,499 & 59,123 & 214,478 \\
11 & - & 28,452 & 86,198 & 72,119 & - \\
12 & - & 43,360 & 78,816 & 43,962 & - \\
13 & 105,430 & - & 119,823 & 53,468 & 193,962 \\
14 & - & 31,148 & 94,363 & 26,317 & 190,936 \\
15 & - & 18,197 & - & 92,249 & 669,293 \\
16 & 6,774 & - & 30,628 & 102,503 & 495,792 \\
17 & 26,696 & - & 90,534 & - & 244,250 \\
18 & - & 23,875 & 54,248 & - & 439,063 \\
19 & - & 21,203 & 48,177 & - & $3,898,640$ \\
20 & - & - & - & 69,008 & 500,673 \\
21 & - & 6,242 & 28,367 & 47,468 & 114,797 \\
22 & - & 4,675 & 21,247 & 35,553 & 85,983 \\
23 & - & 5,691 & - & - & 313,956 \\
24 & - & 3,897 & - & - & 214,997 \\
25 & - & $-11,369$ & - & 92,019
\end{tabular}

Costo ponderado de la atención de 100 pacientes en 25 años* $7,067,674.42$

Costo promedio ponderado de la atención de un paciente en 25 ańos* 70,677

*Los costos se expresan en dólares estadounidenses. Tipo de cambio 16.0772.

** La ponderación incluye tasa de inflación, letalidad y porcentaje de permanencia en cada estadio. 


\section{Discusión}

La enfermedad renal crónica secundaria a diabetes mellitus tipo 2 es un problema de salud que impacta en la población y en los sistemas de salud. Puede ser estudiada desde diferentes aristas, la importancia de abordarla desde la perspectiva del costo por estadio e identificar el costo promedio, estriba en la necesidad de disponer de información útil en la planeación de los sistemas de salud, en ello radica la importancia del trabajo que aquí se presenta.

Utilizar para el diagnóstico del estadio de la enfermedad renal crónica la ecuación de Cockcroft-Gault es un acierto del trabajo ya que es un índice aceptado en la práctica clínica para evaluar la función renal. Aunado a ello haber realizado el diagnóstico por parte del grupo de investigación da certeza y consistencia al diagnóstico.

Sin embargo se tendrá que reconocer que la población estudiada por ser beneficiaria de una institución de seguridad social, posee características que la pueden hacer diferente del resto de la población, y que el perfil de uso de los servicios de salud empleado como referente para calcular el costo, y la propia historia natural de la enfermedad, pueden diferenciarse de otra población a la que se pretenda extrapolar los resultados. No obstante lo anterior, los resultados aquí presentados se constituyen como un referente.

El tiempo de evolución reportado en cada uno de los estadios coincide con lo señalado en la literatura y reafirma el concepto de que a mayor tiempo de evolución mayor daño renal; también se puede apreciar que a mayor daño renal, mayor autocontrol, tal y como lo demuestran las cifras de glucosa en los estadios 4 y 5 (MARTÍNEZ, 2009).

En este trabajo el costo de la atención se incrementa conforme el estadio avanza, alcanzando la máxima expresión en el estadio 5, con semejanzas entre el estadio 1 y 2, al igual que entre el estadio 3 y 4 , escenario que tiene una explicación clínica, ya se ha identificado que en el estadio 5 la necesidad de diálisis en cualquiera de sus modalidades y las complicaciones propias, incrementan ostensiblemente los insumos y en consecuencia el costo.

No obstante al comparar el costo encontrado con lo reportado por la literatura, las diferencias se hacen evidentes (ARREDONDO; RANGEL; DE ICAZA, 1998; GÓRRIZ, 2008). Es verdad que el manejo del paciente con enfermedad 
renal crónica se encuentra perfectamente definido desde la perspectiva clínica, ejemplo de ello son las guías de práctica clínica (SECRETARÍA DE SALUD, 2009), el problema del costeo no reside en la parte clínica.

El problema del costeo tiene dos aristas, por un lado la ausencia de consenso entre los investigadores responsables de calcular los costos, entre estos personajes no se encuentran estandarizados los servicios e insumos que se deben contemplar para identificar costos, de tal suerte que permita la comparación entre los diferentes estudios. De igual forma se deberá estar claro que en el contexto de la economía de la salud, las diferencias de costos del mismo proceso parecieran ser una característica, económicamente se afirma que cada empresa maneja un costo diferente en función de la eficiencia o ineficiencia en el manejo de los recursos, aunado a ello se debe reconocer que el costo es una herramienta con la que la empresa sale al mercado a enfrentar la competencia y obtener poder de mercado y desde esta perspectiva los costos tendían que ser distintos.

Pero más allá de lo anterior, en la medida que la población en el estadio 5 se incremente, los recursos asignados a la salud serán permanentemente insuficientes para cubrir las demandas de atención (CUSUMANO, 2007).

La proyección del costo de la enfermedad renal crónica a lo largo de la vida evidencia la cantidad de recursos que se requieren para brindar atención en el presente y a futuro. Este valor extrapolado al total de la población presenta un panorama lúgubre que puede llevar a los sistemas de salud al caos. Ante este escenario se hace urgente que los sistemas de salud rediseñen las políticas públicas que impidan la progresión de la enfermedad y el avance a estadios superiores, procurando con ello el uso más eficiente de los recursos y el mejor nivel de salud de la población. ${ }^{1}$

\section{Referencias}

ALCÁZAR, R. et al. Sociedad Espańola de Nefrología. Sociedad Española de Medicina Familiar y Comunitaria. Documento de consenso sobre la enfermedad renal crónica. Nefrología, n. 3, p. 273-82, 2008.

AMERICAN DIABETES ASSOCIATION (ADA). Standard Medical Care in Diabetes. Diabetes Care, v. 34, n. 1, p. 11-47, 2012.

ARREDONDO, A.; DAMIAN, T.; DE ICAZA, E. Una aproximación al estudio de costos de servicios de salud en México. Salud Pública Mex, v. 37, p. 437-445, 1995. 
ARREDONDO, A.; RANGEL, R.; DE ICAZA, E. Costo-efectividad de intervenciones para insuficiencia renal crónica terminal. Salud Pública Mex, v. 32, n. 6, p. 556-65, 1998.

CUSUMANO A. Enfermedad renal crónica: necesidad de implementar programas para su detección precoz y prevención de su progresión. Sociedad Latinoamericana de Nefrología e Hipertensión, v. 5, n. 4, p. 139-146, 2007.

GARRIDO, F. et al. Información para rendición de cuentas. Salud México 2006.. México, D.F.: Secretaría de Salud, 2007. Disponible en: http://www.dged.salud.gob.mx/contenidos/ dedss/descargas/rcs/sm2006.pdf>. Acceso: 10 julio 2015.

GARRIDO, F. et al. Rendición de cuentas en salud 2008. México: 2009.p. 25-31. Disponible en: <http://www.dged.salud.gob.mx/contenidos/dedss/descargas/rcs/rcs2008.pdf>. Acceso: 15 julio 2015.

GÓRRIZ, J.; OTERO, A. Impacto socio sanitario de la enfermedad renal crónica avanzada. Nefrología, supl. 3, p. 7-15, 2008.

GRACÍA, S. et al. Documento de consenso: Recomendaciones sobre la utilización de ecuaciones para la estimación del filtrado glomerular en adultos. Sociedad Española de Bioquímica Clínica y Patología Molecular y Sociedad Española de Nefrología. Nefrología, n. 6, p. 658-65, 2006.

INSTITUTO MEXICANO DEL SEGURO SOCIAL. Guía de práctica clínica. Diagnóstico y tratamiento de la diabetes mellitus 2 en el primer nivel de atención. 2009, p. 11-24. Disponible en: <http://www.imss.gob.mx/profesionales/guiasclinicas/Documents/ GER_DiabetesMellitusTipo2.pdf>. Acceso: 3 julio 2015.

Guía de práctica clínica. Prevención, diagnóstico y tratamiento de la Enfermedad Renal Crónica Temprana. Secretaría de Salud. 2009, p. 7-15. Disponible en: http://www. cenecet.salud.gob.mx/interior/gpc.html>. Acceso: 13 julio 2015.

LEVEY, A. S. et al. Enfermedad renal crónica como problema global en Salud Pública: Abordajes e iniciativas propuesta de la Kidney Disease Improving Global Outcomes. Conferencia KDIGO 2007. Kidney Int., n. 3, p. 232-245, 2007.

LEVEY, A. S. et al. A new equation to estimate GFR from serum creatinine: Improved accuracy and updated estimates of prevalence of chronic kidney disease in the United States. Renal Week 2008. American Society Nephrology, 2008.

MARTÍNEZ, A. et al. Estrategias en salud renal: un proyecto de la Sociedad Española de Nefrología. Nefrología, v. 29, n. 3, p. 185-192, 2009.

NATIONAL KIDNEY FOUNDATION. K/DOQI clinical practice guidelines for chronic kidney disease: evaluation, classification and stratification. Am J Kidney Dis., v. 39, supl. 1, p. S1-S266, 2002. 
NATIONAL KIDNEY FOUNDATION. KDOQI clinical practice guidelines and clinical practice recommend dations for anemia in chronic kidney disease. Am J Kidney Dis., v. 47, supl. 3, p. S1-S145, 2006.

SECRETARÍA DE SALUD. Guía de práctica clinica prevención, diagnóstico y tratamiento de la enfermedad renal crónica temprana. México, Secretaría de Salud, 2009. Disponible en: <www.cenetec.salud.gob.mx/interior/gpc.html>. Acceso: 1 ago. 2017.

\section{Nota}

${ }^{1}$ La contribución de cada autor al artículo fue: E. V. Ríos - elaboración de protocolo, análisis de la información, redacción y revisión del texto. R. P. Anaya - elaboración de protocolo, trabajo de campo, análisis de la información, redacción del texto. E. R. V. Daza y L. M. González - elaboración de protocolo, trabajo de campo, redacción y revisión del texto. L. G. RODRÍGUEZ - elaboración de protocolo, construcción de la base de datos, análisis de la información, redacción del texto 


\section{Abstract}

Cost per stage of comprehensive care of type 2 diabetic patients with chronic kidney disease

Objective: To identify the cost of chronic kidney disease by stage in patients with diabetes. Methods: Cost study in type 2 diabetics, in five groups defined from the stage of chronic kidney disease. Those with more than five years of evolution of type 2 diabetes were included; the sample size was calculated for each stage and the selection was simple random. The diagnosis by stage was calculated using the Cockcroft-Gault equation. The average cost was integrated from the profile of use and the unit cost, estimation of the projection of the cost was made, an estimate of the cost was also made for a patient with 25 years old with chronic kidney disease. Results: In stage 1 , the annual cost is $\$ 587.39$, and in stage $5, \$ 10,748.51$. Caring for 25 years in a population of 100 diabetic patients adjusted for lethality, keeping the stadium and inflation costs $\$ 7,067,674$, and the average cost of an individual in this same scenario is $\$ 70,672$. Conclusion: It can be said that the cost per stage of chronic kidney disease in the diabetic patient is high, with marked difference between the different stages.

> Keywords: diabetes mellitus; chronic kidney failure; cost. 


\section{Custo por estágio de cuidados integrais de pacientes diabéticos de tipo 2 com doença renal crônica}

Objetivo: Identificar o custo da doença renal crônica por estágio em pacientes com diabetes. Métodos: estudo de custo em diabéticos de tipo 2, em cinco grupos definidos a partir do estágio de doença renal crônica. Aqueles com mais de cinco anos de evolução do diabetes tipo 2 foram incluídos; o tamanho da amostra foi calculado para cada estágio e a seleção foi aleatória simples. O diagnóstico por fase foi calculado utilizando a equação de CockcroftGault. O custo médio foi integrado a partir do perfil de uso e do custo unitário; foi feita uma estimativa da projeção do custo; e uma estimativa do custo também foi feita para um paciente com 25 anos com doença renal crônica. Resultados: $\mathrm{Na}$ fase 1, o custo anual é de US \$ 587,39 , e na etapa $5, \$ 10,748.51$. O cuidado por 25 anos em uma população de 100 pacientes diabéticos ajustados pela letalidade, mantendo o estádio e a inflação, custa US \$ 7.067.674, e o custo médio de um indivíduo neste mesmo cenário é de US \$ 70.672. Conclusáo: pode-se dizer que o custo por estágio da doença renal crônica no paciente diabético é alto, com diferença acentuada entre os diferentes estágios.

> Palavras-chave: diabetes mellitus; doença renal crônica; custos. 